available for those who consider the present rules to be unreasonably restrictive when applied to their own craft and would regularize the position of navigators who, for safety reasons, already make such manœuvres under the not very satisfactory cover of Rule 27. After a period of use it might be that the permissive actions I have suggested would commend themselves to more and more navigators and that only a minority would continue to maintain course and speed when in the position of the privileged vessel. At this stage consideration could well be given to making such actions compulsory for all vessels on the lines of Commander Clissold's proposals.

Mariners are by nature and very properly cautious people and changes should therefore be made in very small steps or, if a large step is unavoidable, then the transition must be allowed to occur slowly. Any radical proposal which does not provide for hysteresis of this sort is unlikely to find acceptance whatever its theoretical merits.

I should emphasize that the points I have raised are only concerned with ways and means. I do not believe that it is practicable to change to Commander Clissold's Rules in one step, but if we could evolve towards them I am sure that they would prove more satisfactory than the present Rules.

\title{
The Pair Rule and the Collision Problem
}

\author{
Rear Admiral J. García-Frías \\ (Spanish Navy)
}

I. INTRODUCTION. In my recent paper $^{1}$ I proposed a set of manœuvring rules intended to solve the operational aspect of the collision problem. These rules are simple, but they are different if the bearing is lesser or greater than $90^{\circ}$. The object of this paper is to present a simpler rule valid for every bearing between $0^{\circ}$ and $180^{\circ}$.

2. The PAIR RULE. Except for the singular situation of both vessels meeting head-on or head-to-stern, every encounter involving danger of collision is characterized by the fact that the heading of both vessels is on the same side of the sight line. Close-quarter situations also involve, in general, those standing head-on, except for some situations with the heading on opposite sides of the sight line when one or both of them heads close to this line. Consequently, the evading manœuvre must be such that both headings are on opposite sides of the sight line and opening enough to ensure a safe passing.

With vessels in sight of one another, because of the aspect it is easy to get the heading of both vessels on opposite sides of the sight line. But aspect does not help in the radar case in an immediate and continuous way. Nevertheless, it is possible to achieve the same objective with the information given directly by radar by keeping to the Pair Rule as follows.

Fig. I presents the steady bearing situation. Since both vectors are on the same side of the sight line, it is easy to establish a convention for one of the vessels to 


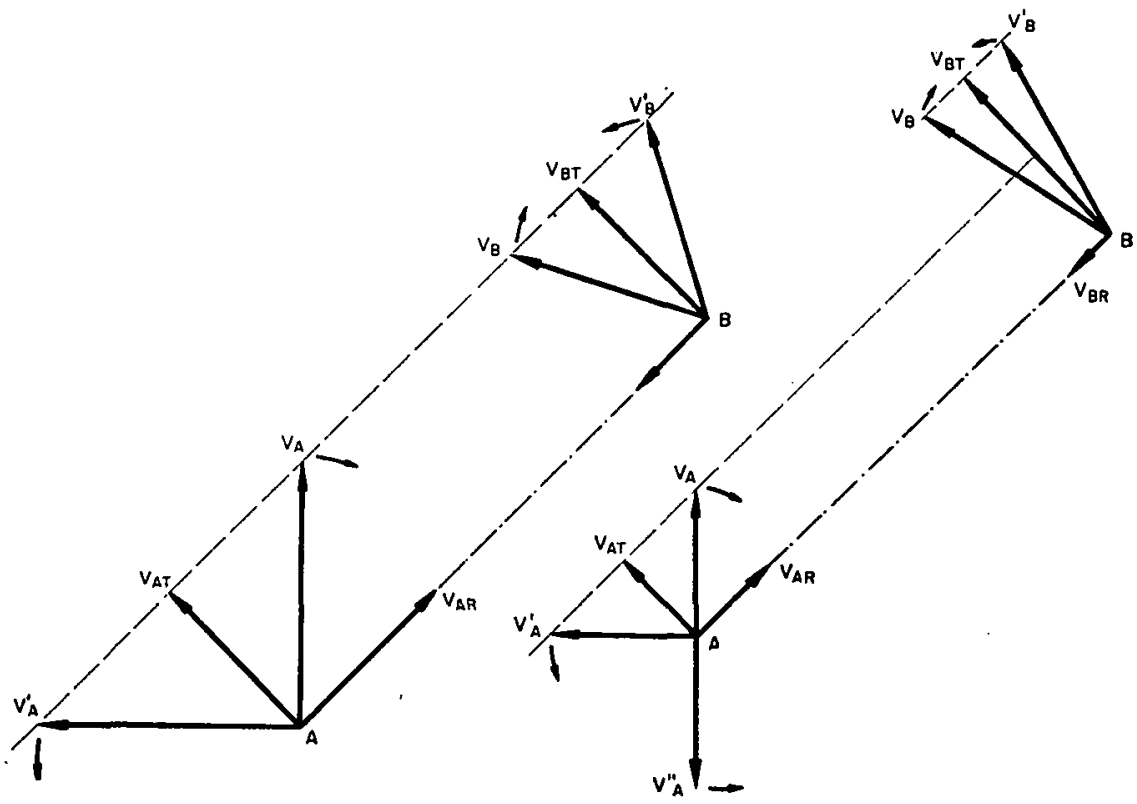

FIGS I AND 2

stand on the same side and the other one to turn towards the other side of the sight line. The convention generally involved in the Rule of the Road for bearings less than $90^{\circ}$-i.e., vector $V_{\mathrm{A}}$ turning towards the other side of the sight line and vector $V_{B}$ remaining on the same side-may be generalized with the use of radar by turning vector $V_{\mathrm{A}}$ in the shortest way towards the other side of the sight line and by keeping vector $V_{B}$ on the same side without any restriction on its course. Consequently, we can derive the following Pair Rule: If the bearing of a vessel echo is steady to starboard, a turn by the shortest direction shall be made towards the other side of the sight line; if the echo is steady to port the heading shall be kept on the same side of the sight line.

Fig. 2 shows the encounter with bearing of vessel B closing to $V_{\mathrm{A}}$ or $V_{\mathrm{A}}^{\prime}$. The normal component $V_{\mathbf{B T}}$ is then greater than the normal component $V_{\mathbf{A T}}$ and, consequently, vessel $A$ will always be sure that vector $V_{B}$ or $V_{B}^{\prime}$ is on the same side of the sight line. With regard to vessel $B$, the bearing of $A$ is opening, but this situation may arise both with normal component $V_{\mathbf{A T}}$ less than normal component $V_{\mathrm{BT}}$ on the same side of the sight line and with normal component $V_{\mathrm{AT}}$ on the other side of this line. Therefore, if vessel A turns towards the other side of the sight line by the shortest way if bearing of vessel B is closing, and this vessel keeps course on the same side of this line if vessel $A$ is opening, it always leads to a pair of vectors in opposite directions, because in the case when normal component $V_{\mathrm{AT}}$ would be on the other side of the sight line, the bearing of $\mathrm{B}$ would also be opening for vessel $A$ and, consequently, she also would keep course at this side of the sight line. The Pair Rule is then as follows: If the bearing of a vessel echo is closing, a turn by the shortest way shall be made towards the other side of the sight line; if the echo is opening, the heading shall be kept on the same side of the sight line. If we reconstruct both rules, we have the following general Pair Rule: 
If the bearing of a vessel echo is steady to starboard or is closing, a turn by the shortest direction shall be made towards the other side of the sight line; if the echo bears steady to port or is opening, the heading shall be kept on the same side of the sight line.

3. Conclusion. This Pair Rule is simpler than the manœuvring rules I have proposed previously ${ }^{1}$, but all of them are simple because they only work out the operational aspect of the collision problem; the organic aspect is solved by the Sector Rule1, which involves the best guarantee of a safe passing.

R E F E R E N C E

1 Garcia-Frias, J. (1965). The sector rule and the collision problem. This Journal, 18 , 141.

\section{The Vinland Map}

\section{R. A. Skelton}

THE January issue of the Journal (pp. I 24-5) contained a summary of Professor E. G. R. Taylor's views on the Vinland Map. To the courtesy of the Editor I owe the opportunity both to read this note in proof and to comment on it in the present issue. I have not seen Professor Taylor's complete study nor her drawings. She expressed some of her misgivings to me in correspondence after (in 1 962) seeing a photocopy of the map and reading in typescript my study of the map, which is included in the recently published monograph.

Professor Taylor's judgment was formed from examination of the map, without knowledge of the two textual documents with which it is associated or of the common physical characteristics which link it to them. That these other documents-the Tartar Relation and a fragment of the Speculum historiale of Vincent of Beauvais--are authentic, and that they were copied (i.e. written) about 1440 , probably in the Upper Rhineland, are conclusions on which no doubt has yet been expressed; nor does it seem likely that any could be seriously sustained. It must however be recognized that, strong as are the arguments (from palaeography, bibliotics and content) that connect the map with these documents, the view that it is a modern counterfeit cannot, in the absence of information on its history, be absolutely disproved. One can even visualize possible methods by which a hypothetical forger might have gone to work to produce such a map.

We must understand clearly what kind of evidence is required to settle the issue of authenticity positively one way or the other. Obviously the history of the map and of the associated textual documents would do so, if and when it comes to light. So too would chemical analysis of the materials (vellum, ink), could this be undertaken without risk. Yale University Library bears the responsibility for preservation of the documents, and (in the words of the foreword to the monograph) 'all [physical] tests that would not involve damage or destruction of the manuscript have been applied'. The fact that these tests, together with analysis of the form and content of the map in the historical 